\title{
Suppressing excitation effects in microwave induced thermoacoustic tomography by multi-view Hilbert transformation
}

Cite as: Appl. Phys. Lett. 110, 053701 (2017); https://doi.org/10.1063/1.4975204

Submitted: 15 December 2016 . Accepted: 18 January 2017. Published Online: 30 January 2017

Yu He, Yuecheng Shen (D), Changjun Liu (D), and Lihong V. Wang

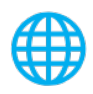

View Online

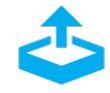

Export Citation

\section{ARTICLES YOU MAY BE INTERESTED IN}

Homogenizing microwave illumination in thermoacoustic tomography by a linear-to-circular polarizer based on frequency selective surfaces

Applied Physics Letters 111, 063703 (2017); https://doi.org/10.1063/1.4993942

Microwave-excited ultrasound and thermoacoustic dual imaging

Applied Physics Letters 110, 183701 (2017); https://doi.org/10.1063/1.4983166

Photoacoustic imaging in biomedicine

Review of Scientific Instruments 77, 041101 (2006); https://doi.org/10.1063/1.2195024

\section{Lake Shore} CRYOTRONICS

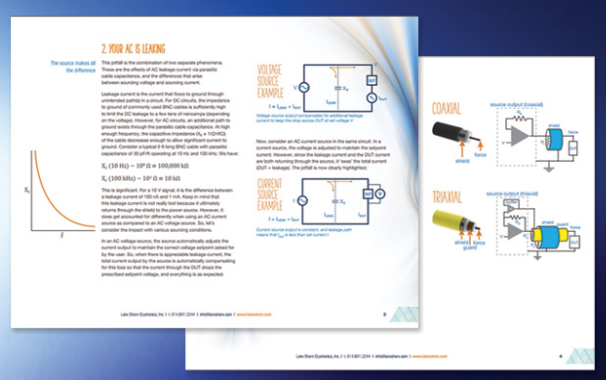

5 Electronic

- Measurement Pitfalls to Avoid

Get the whitepaper $\bullet$ 


\title{
Suppressing excitation effects in microwave induced thermoacoustic tomography by multi-view Hilbert transformation
}

\author{
Yu He, ${ }^{1,2}$ Yuecheng Shen, ${ }^{1}$ Changjun Liu, ${ }^{2, a)}$ and Lihong V. Wang ${ }^{1, a)}$ \\ ${ }^{1}$ Optical Imaging Laboratory, Department of Biomedical Engineering, Washington University in St. Louis, \\ St. Louis, Missouri 63130, USA \\ ${ }^{2}$ School of Electronics and Information Engineering, Sichuan University, Chengdu 610041, China
}

(Received 15 December 2016; accepted 18 January 2017; published online 30 January 2017)

\begin{abstract}
Microwave induced thermoacoustic tomography (TAT) images usually suffer from distortions arising from the microwave polarization effect and standing wave effect. The microwave polarization effect, resulting from linearly polarized microwave illumination, splits the image of the object along the polarization direction, while the standing wave effect, when the object size is larger than the microwave wavelength within the object, modulates the image of the object. Both effects cause non-uniform energy distribution in a uniformly absorbing object and create artifacts in the reconstructed images. To address these problems in TAT, we propose an image reconstruction method that combines multi-view Hilbert transformation with the back-projection algorithm. We experimentally validate this method by imaging breast and brain tumor phantoms, showing that the aforementioned distortions are significantly suppressed. We anticipate that this method will contribute to clinical tumor diagnosis. Published by AIP Publishing.
\end{abstract}

[http://dx.doi.org/10.1063/1.4975204]

Microwave induced thermoacoustic tomography (TAT), combining the high contrast of microwave imaging and high resolution of ultrasound imaging, is a potential nonionizing candidate for breast tumor diagnosis. ${ }^{1-8}$ There are considerable differences in the microwave dielectric properties of malignant tissue and normal adipose-dominant tissue in the breast. ${ }^{9}$ When a morbid breast is irradiated by a microwave pulse, the malignant tissue, which has a higher dielectric loss, absorbs more energy and creates stronger thermoacoustic waves than the surrounding healthy tissue. The generated thermoacoustic waves carrying information about the microwave absorption distribution of the breast can be measured by a single-element ultrasonic transducer or a transducer array. Besides its application in breast imaging, TAT also shows potential for brain imaging, ${ }^{10-12}$ and it is a candidate for brain tumor detection.

The absorbed microwave power density is proportional to the product of the local tissue loss and the squared amplitude of the electric field within the irradiated medium. ${ }^{13}$ Since the reconstructed images in TAT are expected to represent the real structure of the tissue, i.e., the distribution of dielectric loss, the tissue should be homogeneously illuminated; otherwise, the images will reveal not only the different dielectric properties but also the inhomogeneous electric field. However, homogenous illumination is difficult to achieve in practice. In the conventional TAT reconstruction process ${ }^{14}$ non-uniform microwave energy deposition in the object distorts the TAT images for two reasons. ${ }^{15-17}$ One is the microwave polarization effect, which occurs when the object is under linearly polarized microwave illumination (e.g., microwave from a pyramidal horn antenna); the other is the microwave standing wave effect inside the object,

\footnotetext{
${ }^{\text {a) }}$ Authors to whom correspondence should be addressed. Electronic addresses: cjliu@ieee.org and lhwang@wustl.edu.
}

which arises when the object is larger than the microwave wavelength within the object, and there is high contrast between the object and background. Both factors cause ambiguities in image interpretation.

Another challenge in the conventional TAT reconstruction process is that the reconstructed images present bipolar (i.e., both positive and negative) pixel values due to the band-pass response of the ultrasonic transducer. ${ }^{18}$ We want to quantify the microwave energy deposition, so nonnegative pixel values are desired. Bipolar images are difficult to interpret because both positive and negative peak values indicate strong microwave absorption. Unipolar images can be obtained by deconvolving the transducer's electrical impulse response. ${ }^{19,20}$ However, this process is usually computationally time consuming.

In order to address the aforementioned problems in conventional TAT reconstruction, we propose an image reconstruction method combining multi-view Hilbert transformation with the back-projection algorithm. After reconstruction, the images become unipolar, and the artifacts created by the polarization effect and standing wave effect are significantly suppressed.

The experimental setup is shown in Fig. 1(a). Microwave pulses transmitted from a microwave generator (peak power: $60 \mathrm{~kW}$, pulse width: $0.6 \mu \mathrm{s}$, frequency: $3 \mathrm{GHz}$, and repetition rate: $10 \mathrm{~Hz}$ ) were delivered to the target through a pyramidal horn antenna $\left(10.8 \times 7.2 \mathrm{~cm}^{2}\right)$. The target absorbed microwave energy and created thermoacoustic waves. The actual averaged microwave power density at the phantom surface was $4.7 \mathrm{~mW} / \mathrm{cm}^{2}$, which is below the IEEE safety standard $\left(10 \mathrm{~mW} / \mathrm{cm}^{2}\right.$ at $\left.3 \mathrm{GHz}\right) .{ }^{21}$ An ultrasonic transducer $(2.25 \mathrm{MHz})$ points horizontally to the rotation center and scans step by step around the phantom. At each step, the induced thermoacoustic signals are received by the transducer, amplified, and transferred to a computer for 


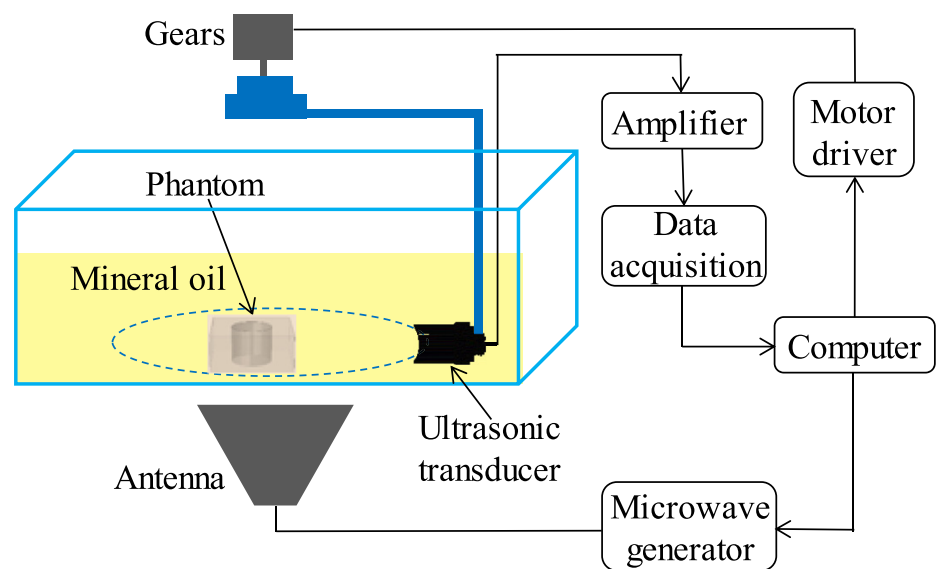

(a)

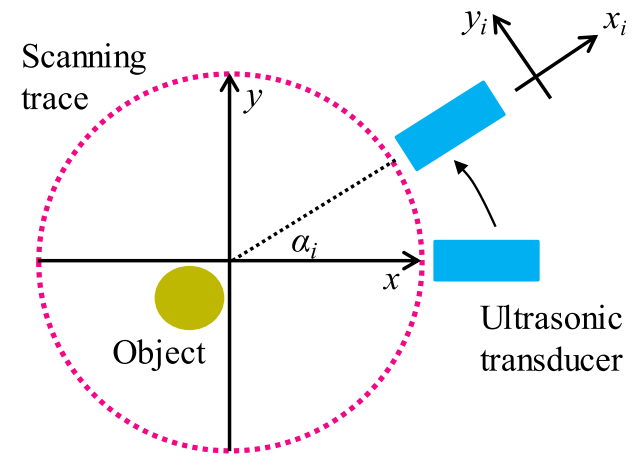

(b)

FIG. 1. (a) Experimental setup. (b) Scanning configuration. The acoustic axis changes with the rotation angle.

image reconstruction. Details of our TAT system have been reported in our previous work. ${ }^{17}$

In this work, multi-view Hilbert transformation is applied to recover the envelope of the image, which converts bipolar images into unipolar images ${ }^{18}$ and effectively suppresses the polarization and standing wave effects. Since Hilbert transformation must be performed along the axis of the time of arrival, i.e., the acoustic axis in the system, the direction of the transformation needs to be altered at each scanning step. Otherwise, the mismatch between the Hilbert transformation direction and the acoustic axis will cause artifacts. Since the transducer rotates around the object, the acoustic axis rotates accordingly. Therefore, two coordinate systems are used during the reconstruction process. As shown in Fig. 1(b), one is the fixed global coordinates $(x, y)$, where $\alpha_{i}$ denotes the azimuth angle of the transducer with respect to the center. The other one is the rotating local coordinates $\left(x_{i}, y_{i}\right)$.

Here, we describe the general scheme of the reconstruction process:

1. At each rotation angle $\alpha_{i}$, we reconstruct the measured acoustic pressure signal $p^{i}(\vec{r}, t)$ by using the filtered backprojection (FBP) algorithm ${ }^{22}$ and thereby obtain the reconstructed TA pressure $p_{0}^{i}(\vec{r})$. The reconstruction formula is as follows:

$$
p_{0}^{i}(\vec{r})=\int_{\Omega}\left[2 p^{i}(\vec{r}, t)-2 t \partial p^{i}(\vec{r}, t) / \partial t\right] d \Omega / \Omega,
$$

where $p^{i}(\vec{r}, t)$ is the acoustic pressure measured by the transducer at the position $\vec{r}$ and time $t$, and the transducer is at the angle $\alpha_{i}$. The term $d \Omega / \Omega$ is a weighting factor. Here, we note that $p_{0}^{i}(\vec{r})$ presents bipolar pixel values.

2 . By rotating $p_{0}^{i}(\vec{r})$ by an angle of $-\alpha_{i}$, we change the global coordinates to the local coordinates. Specifically, the pixel coordinates in these two coordinate systems are connected by the following matrix equation:

$$
\left(\begin{array}{l}
x_{i} \\
y_{i}
\end{array}\right)=\left(\begin{array}{cc}
\cos \alpha_{i} & \sin \alpha_{i} \\
-\sin \alpha_{i} & \cos \alpha_{i}
\end{array}\right)\left(\begin{array}{l}
x \\
y
\end{array}\right) .
$$

After transforming all the pixel coordinates, the image must be resampled using a square grid of pixel values. We apply bilinear interpolation, which determines the value based on the weight of the four nearest pixels and thereby acquire $p_{1}^{i}(\vec{r}): p_{1}^{i}(\vec{r})=R_{-\alpha_{i}}\left[p_{0}^{i}(\vec{r})\right]$, where $R$ is the rotating operator.

3. By taking the absolute value of the Hilbert transform of the signal $p_{1}^{i}(\vec{r})$, we recover the envelope of the signals and acquire $p_{2}^{i}(\vec{r}): p_{2}^{i}(\vec{r})=\operatorname{Abs}\left\{H\left[p_{0}^{i}(\vec{r})\right]\right\}$, where $\mathrm{Abs}$ is the absolute value operator and $H$ is the Hilbert transform operator. The pixel values change from bipolar to unipolar.

4. By rotating the signal $p_{2}^{i}(\vec{r})$ back by an angle of $\alpha_{i}$, the local coordinate system is transformed back to the global coordinate system, and we acquire $p_{3}^{i}(\vec{r}): p_{3}^{i}(\vec{r})=R_{\alpha_{i}}\left[p_{2}^{i}(\vec{r})\right]$.

5. We repeat steps 1 to 4 for all angles and average all the acquired images to obtain the final reconstructed image $p(\vec{r})$. The flow chart of the reconstruction process is shown in Fig. 2, and the whole process can be formulated as

$$
p(\vec{r})=\frac{1}{N} \sum_{i=1}^{N} R_{\alpha_{i}}\left\{\operatorname{Abs}\left\{H\left\{R_{-\alpha_{i}}\left[p_{0}^{i}(\vec{r})\right]\right\}\right\} .\right.
$$

To reconstruct an image with $250 \times 250$ pixels, we estimated that the proposed algorithm with multi-view Hilbert transformation took about $9.2 \mathrm{~s}$ on our personal computer, which was about 3 times the computational time required by the conventional FBP ( $3.3 \mathrm{~s}$ on the same computer). We note that the extra time consumed was mainly occupied by processing the interpolation during matrix rotation.

Using this reconstruction scheme, we validated our proposed method by imaging tumor phantoms. The breast tumor phantoms were made of porcine fat with a central hole filled with a water-based gel, as shown in Fig. 3. The gel, made of $3 \%$ agar and $97 \%$ water, mimicked a breast tumor, and the surrounding porcine fat mimicked normal breast tissue. Considering that the wavelength of a $3 \mathrm{GHz}$ microwave within the tumor phantom is about $12 \mathrm{~mm}$, we prepared two $10 \mathrm{~mm}$ tall cylindrical tumor phantoms with diameters of $10 \mathrm{~mm}$ and $15 \mathrm{~mm}$ to illustrate the polarization and standing wave distortions, respectively.

We first studied the suppression of the microwave polarization effect by applying our proposed multi-view Hilbert transformation. We used a pyramidal horn antenna, radiating linearly polarized microwaves, to illuminate the tumor 

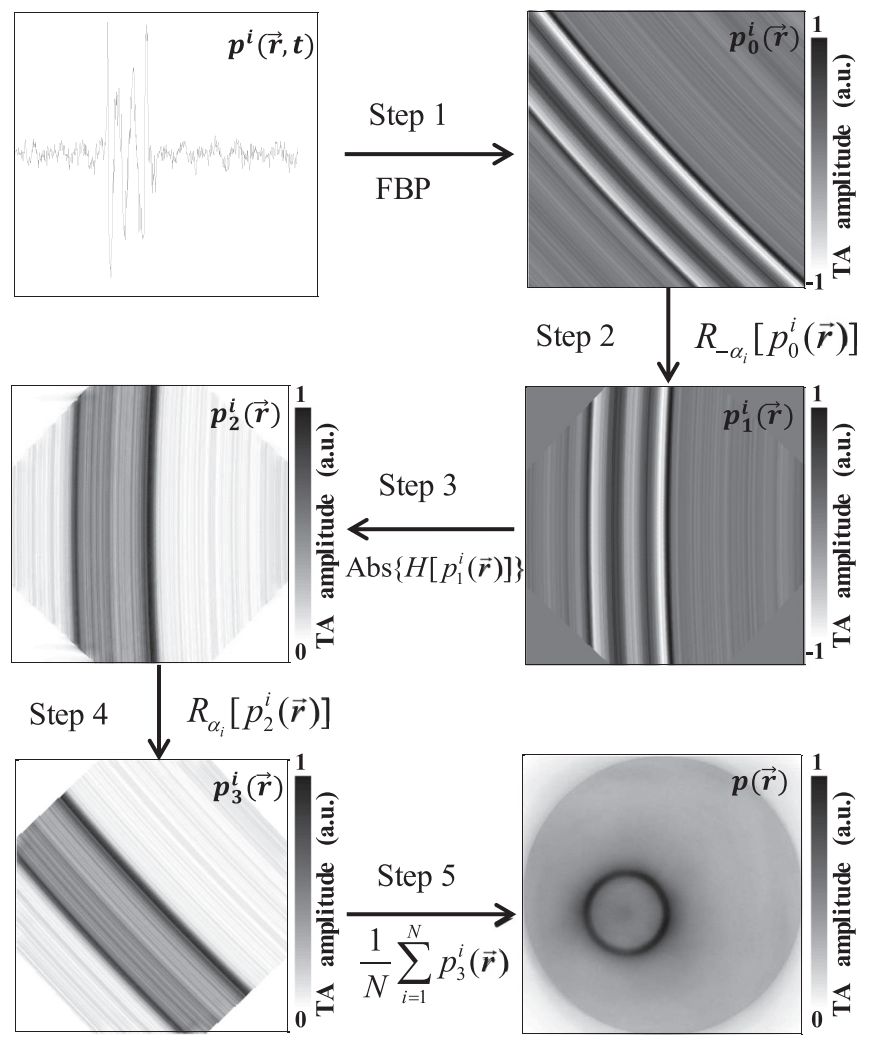

FIG. 2. Flow chart of the reconstruction process.

phantom in Fig. 3(a). The polarization direction of the horn antenna was along the $y$ direction. The microwave absorption of the tumor phantom was simulated by COMSOL finite element software, with the result shown in Fig. 4(a). The microwave absorption in the phantom is not homogeneously distributed: it is stronger along the polarization direction and weaker normal to the polarization direction. In the original FBP algorithm, the reconstructed image is a direct reflection of the microwave energy absorption in the object. Therefore, like the simulated results, the tumor phantom splits into two parts in the reconstructed image, as shown in Fig. 4(b). After applying the multi-view Hilbert transformation, the "split" pattern in the reconstructed image is well suppressed, as shown in Fig. 4(c), which makes the image more realistic.

Another image distortion in TAT results from the microwave standing wave effect. As shown in Fig. 3(b), when the diameter of the tumor (here, $15 \mathrm{~mm}$ ) is larger than the microwave wavelength in the tumor $(12 \mathrm{~mm})$, the large dielectric

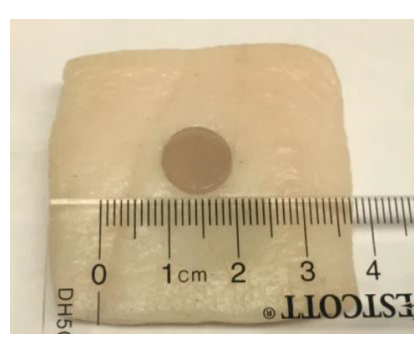

(a)

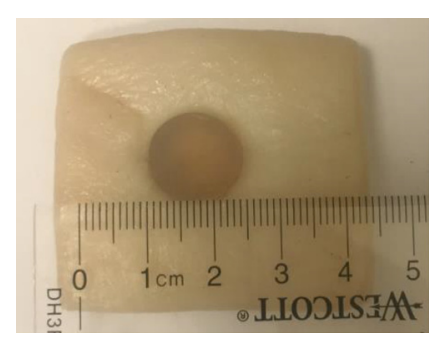

(b)
FIG. 3. Experimental breast tumor phantoms made of porcine fat with a small hole at the center filled with either a $10 \mathrm{~mm}$ (a) or a $15 \mathrm{~mm}$ (b) diameter cylindrical agar gel.

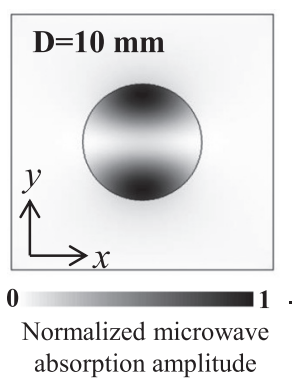

(a)

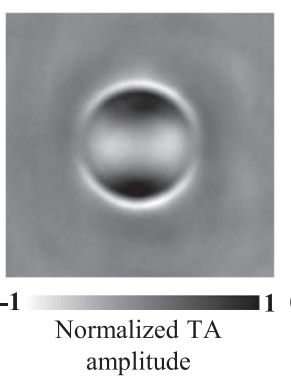

(b)

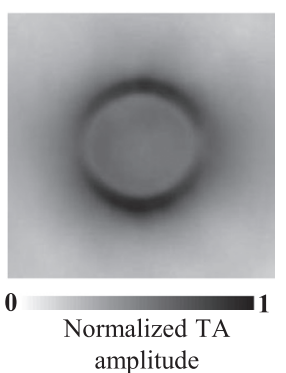

(c)
FIG. 4. Tumor phantom made of a $10 \mathrm{~mm}$ diameter cylindrical agar gel under linearly polarized illumination. (a) Simulated microwave absorption of the tumor phantom. (b) Bipolar image reconstructed by using the FBP algorithm. (c) Unipolar image reconstructed by applying the multi-view Hilbert transformation.

constant of the tumor causes a standing wave effect, ${ }^{23}$ giving a non-uniform microwave absorption distribution. Fig. 5 presents the simulated microwave absorption and reconstructed results of both linearly and circularly polarized cases. In the linearly polarized case, the simulated microwave absorption and the reconstructed image both show periodical patterns along the $x$ direction. In the circularly polarized case, we used a helical antenna to illuminate the tumor phantom. Unlike the pyramidal horn antenna, in which the electric field at a fixed point in space remains pointing in a fixed direction, the helical antenna radiates microwaves in both the $x$ direction and $y$ direction, as well as in every plane in between. Although the boundaries of the tumor are homogeneous in the simulated microwave absorption and the reconstructed image, the microwave absorption at the center of tumor is heterogeneous and looks like three concentric circles. After applying multi-view

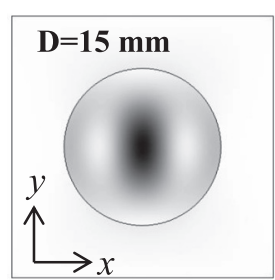

0 Normalized microwave absorption amplitude

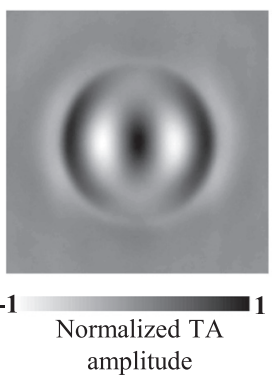

amplitude

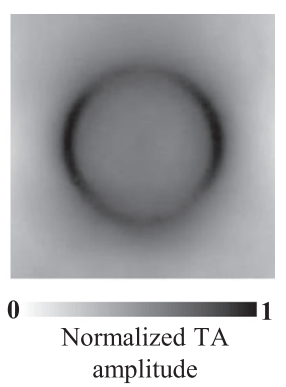

amplitude (a) Linearly polarized illumination
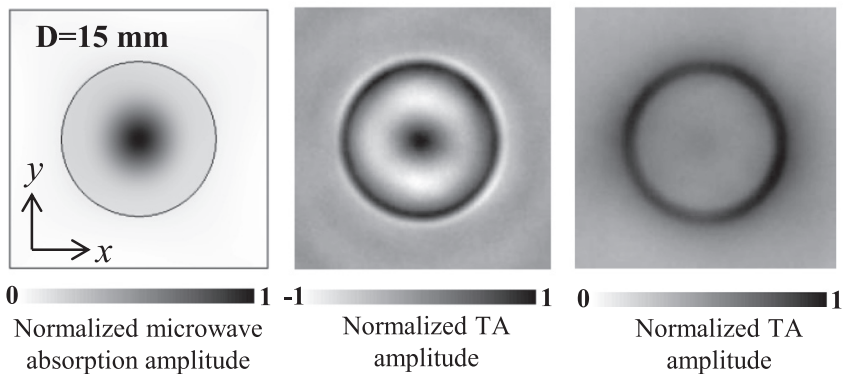

(b) Circularly polarized illumination

FIG. 5. Tumor phantom made of a $15 \mathrm{~mm}$ diameter cylindrical agar gel inside porcine fat under (a) linearly polarized illumination and (b) circularly polarized illumination. The left panel shows the simulated microwave absorption of the tumor phantom. The middle panel shows bipolar images reconstructed by using the FBP algorithm. The right panel shows unipolar images reconstructed by applying the multi-view Hilbert transformation. 


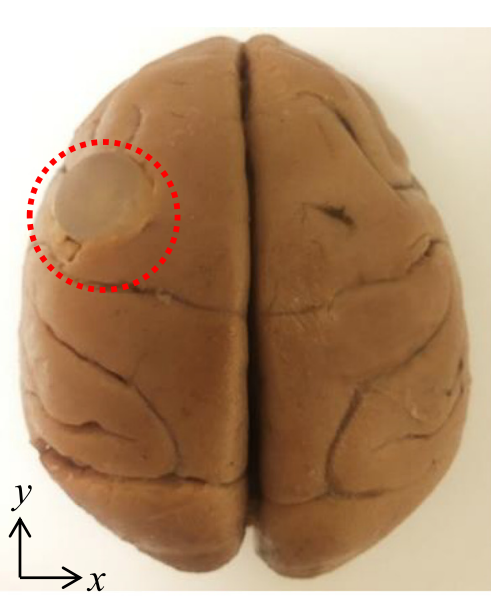

(a)

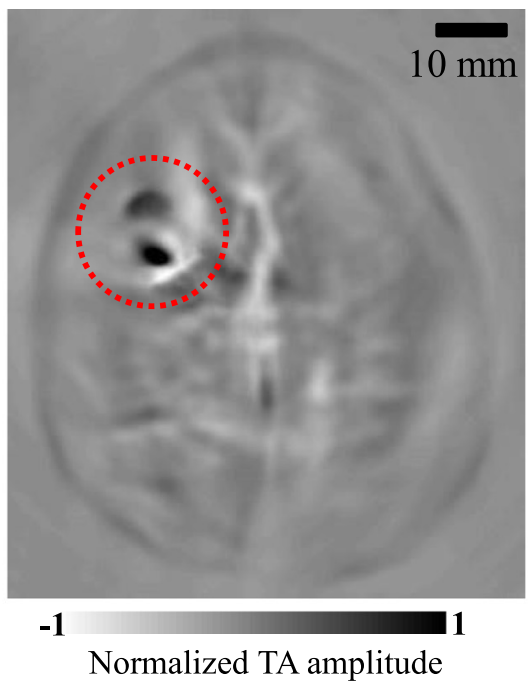

(b)

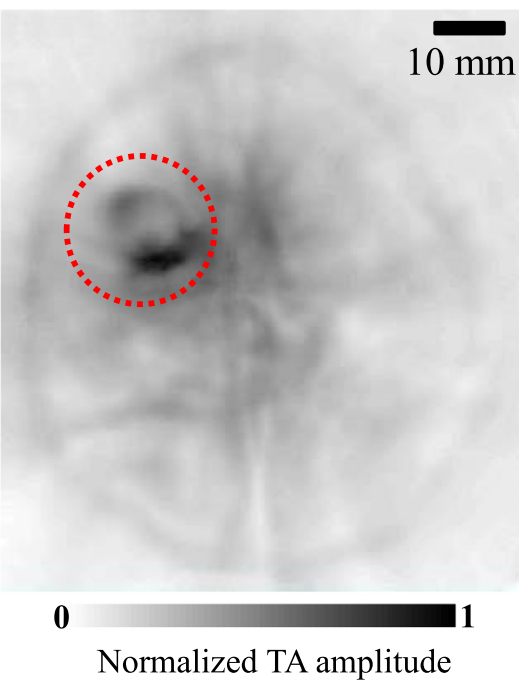

(c)

FIG. 6. (a) Monkey brain tumor phantom with a $10 \mathrm{~mm}$ diameter cylindrical agar gel. (b) Bipolar image reconstructed by using the FBP algorithm. (c) Unipolar image reconstructed by applying the multi-view Hilbert transformation.

Hilbert transformation, the boundaries of the tumor are strengthened, while the artifacts induced by the standing wave effect are significantly suppressed.

In the breast tumor phantom, the dielectric contrast between the tumor (conductivity $\sigma \approx 3 \mathrm{~S} / \mathrm{m}$ and relative permittivity $\left.\varepsilon_{\mathrm{r}} \approx 70\right)$ and the porcine fat $(\sigma \approx 0.2 \mathrm{~S} / \mathrm{m}$ and $\left.\varepsilon_{\mathrm{r}} \approx 5\right)$ is high. Thus, the porcine fat absorbs much less microwave energy than the tumor does. Although the absorbed microwave energy perpendicular to the microwave polarization direction is weak, the boundaries along the $x$ direction can still be distinguished from the porcine fat background in Fig. 4(b).

However, using TAT to detect brain tumors poses a different problem: the dielectric contrast between the tumor and the normal brain tissue $\left(\sigma \approx 2.3 \mathrm{~S} / \mathrm{m}\right.$ and $\left.\varepsilon_{\mathrm{r}} \approx 55\right)$ is not high, which makes the boundaries of the tumor perpendicular to the polarization direction indiscernible. To address this concern, we did another experiment with a monkey brain tumor phantom. A $10 \mathrm{~mm}$ diameter agar gel mimicking a tumor was placed inside an ex vivo monkey brain, as shown in Fig. 6(a). In the conventional FBP reconstructed result, the boundaries of the tumor along the $x$ direction cannot be distinguished from the normal brain tissue. The image of the tumor splits into two parts along the polarization direction, and the shape is totally distorted, as shown in Fig. 6(b). When the proposed multi-view Hilbert transformation was performed, the boundaries of the tumor were well revealed, and the shape and size of the tumor were better presented, as shown in Fig. 6(c). Further, in the unipolar image, the amplitude of the signals from normal brain tissue is weakened, and the tumor becomes more noticeable in the unipolar image due to its stronger microwave absorption.

We note that the microwave polarization effect may also be suppressed by using a circular polarized antenna, ${ }^{17}$ e.g., a helical antenna, to illuminate the tumor, or by near-field imaging using magnetically resonant coils. ${ }^{24-26}$ Since the standing wave effect occurs due to the comparable size of the tumor size and the microwave wavelength, increasing the microwave wavelength is another way to suppress the standing wave effect. However, the dimensions of the antenna would increase accordingly, which would enlarge the TAT system.

To conclude, in this work, we proposed a multi-view Hilbert transformation method combined with the back projection algorithm to suppress the microwave polarization effect and standing wave effect in microwave induced thermoacoustic tomography. Moreover, unipolar images are obtained with strengthened tumor boundaries in the images. Thus, images reconstructed using the proposed multi-view Hilbert transformation can potentially help physicians locate the position and determine the size of a tumor more accurately. Our method represents an important step towards clinical tumor detection in the breast and brain.

This work was supported in part by the China 973 program 2013CB328902 and the China Scholarship Council.

${ }^{1}$ L. V. Wang, X. Zhao, H. Sun, and G. Ku, Rev. Sci. Instrum. 70(9), 3744 (1999).

${ }^{2}$ R. A. Kruger, K. D. Miller, H. E. Reynolds, W. L. Kiser, Jr., D. R. Reinecke, and G. A. Kruger, Radiology 216(1), 279 (2000).

${ }^{3}$ L. Nie, D. Xing, Q. Zhou, D. Yang, and H. Guo, Med. Phys. 35(9), 4026 (2008).

${ }^{4}$ X. Wang, D. R. Bauer, R. Witte, and H. Xin, IEEE Trans. Biomed. Eng. 59(10), 2782 (2012).

${ }^{5}$ X. Wang, T. Qin, R. S. Witte, and H. Xin, IEEE Transactions on Microwave Theory Tech. 63(5), 1489 (2015).

${ }^{6}$ F. Ye, Z. Ji, W. Ding, C. Lou, S. Yang, and D. Xing, IEEE Trans. Med. Imaging 35(3), 839 (2016).

${ }^{7}$ Y. Fu, Z. Ji, W. Ding, F. Ye, and C. Lou, Med. Phys. 41(11), 110701 (2014).

${ }^{8}$ X. Zhu, Z. Zhao, J. Wang, J. Song, and Q. Liu, IEEE Trans. Biomed. Eng. 60(5), 1298 (2013).

${ }^{9}$ M. Lazebnik, L. McCartney, D. Popovic, C. B. Watkins, M. J. Lindstrom, J. Harter, S. Sewall, A. Magliocco, J. H. Booske, and M. Okoniewski, Phys. Med. Biol. 52(10), 2637 (2007).

${ }^{10}$ Y. Xu and L. V. Wang, IEEE Trans. Ultrason., Ferroelectr., Freq. Control 53(3), 542 (2006).

${ }^{11}$ P. Stefanov and G. Uhlmann, Inverse Probl. 27(4), 045004 (2011).

${ }^{12}$ J. Yao and L. V. Wang, Neurophotonics 1(1), 011003 (2014).

${ }^{13}$ L. Huang, L. Yao, L. Liu, J. Rong, and H. Jiang, Appl. Phys. Lett. 101(24), 244106 (2012). 
${ }^{14}$ M. Xu and L. V. Wang, Med. Phys. 29(8), 1661 (2002).

${ }^{15}$ C. Li, M. Pramanik, G. Ku, and L. V. Wang, Phys. Rev. E 77(3), 031923 (2008).

${ }^{16}$ M. Pramanik, G. Ku, C. Li, and L. V. Wang, Med. Phys. 35(6), 2218 (2008).

${ }^{17}$ Y. He, C. Liu, L. Lin, and L. V. Wang, IEEE Antennas Wireless Propag. Lett. PP(99), 1-1 (2017).

${ }^{18}$ G. Li, L. Li, L. Zhu, J. Xia, and L. V. Wang, J. Biomed. Opt. 20(6), 066010 (2015).

${ }^{19}$ J. Zhang, M. A. Anastasio, P. J. La Rivière, and L. V. Wang, IEEE Trans. Med. Imaging 28(11), 1781 (2009).
${ }^{20}$ C. Huang, K. Wang, L. Nie, L. V. Wang, and M. A. Anastasio, IEEE Trans. Med. Imaging 32(6), 1097 (2013).

${ }^{21}$ IEEE Std C95.1TM, IEEE Standard for Safety Levels with Respect to Human Exposure to Radio Frequency Electromagnetic Fields, $3 \mathrm{kHz}$ to $300 \mathrm{GHz}$ (IEEE, 2005).

${ }^{22}$ M. Xu and L. V. Wang, Phys. Rev. E 71(1), 016706 (2005).

${ }^{23}$ P. S. Tofts, J. Magn. Reson., Ser. B 104(2), 143 (1994).

${ }^{24}$ F. Gao, Y. Zheng, X. Feng, and C.-D. Ohl, Appl. Phys. Lett. 102(6), 063702 (2013).

${ }^{25}$ X. Feng, F. Gao, and Y. Zheng, Appl. Phys. Lett. 103(8), 083704 (2013).

${ }^{26}$ X. Feng, F. Gao, R. Kishor, and Y. Zheng, Sci. Rep. 5, 11489 (2015). 